\title{
Contribution of Presepsin, Procalcitonin and C-reactive protein to the SOFA Score in Early Sepsis Diagnosis in Emergency Abdominal Surgical Patients
}

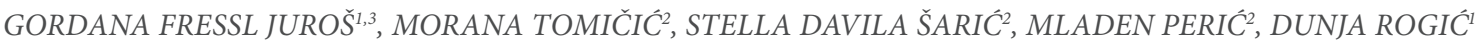

1 Department of Laboratory Diagnostics, University Hospital Centre Zagreb, Kišpatićeva 12, Zagreb, Croatia

2 Department of Anesthesiology and ICU, University Hospital Centre Zagreb, Kišpatićeva 12, Zagreb, Croatia

3 Children's Hospital Srebrnjak, Srebrnjak 100, Zagreb, Croatia

\author{
Corresponding author \\ Gordana Fressl Juroš \\ Children's Hospital Srebrnjak, \\ Srebrnjak 100, 10000 Zagreb, Croatia \\ E-mail: gordana.fressl@zg.t-com.hr \\ Tel.: +38598432681
}

\section{ABSTRACT}

Purpose: This study examined whether the addition of biomarkers presepsin (PSEP), procalcitonin (PCT) and C-reactive protein (CRP) to the initial SOFA (iSOFA) score can improve diagnostic accuracy of early sepsis diagnosis in emergency abdominal surgery patients.

Materials and Methods: Seventy-two study subjects had diagnosis of acute abdomen due to gastrointestinal disturbances. The study evaluated diagnostic accuracy and predictive value of two models (iSOFA only and iSOFA combined with three biomarkers) for sepsis diagnosis.

Results: The AUC value for the iSOFA was highest, followed by the AUC value obtained for PSEP, PCT and CRP (0.989, $0.738,0.694$ and 0.606 , respectively). The logistic regression analysis of the two models showed for the first model that patients with a higher iSOFA score are almost two times more likely to suffer from sepsis. In the second model, patients with a higher iSOFA score and a higher level of biomarkers are three times more likely to have sepsis.

Conclusions: Although the SOFA score is known to be the best diagnostic tool for sepsis diagnosis, it seems that among the three investigated markers PSEP and PCTalthough not contributing to the iSOFA score- are good independent markers with significantly higher levels in septic than in non-septic patients. PSEP has the highest diagnostic accuracy for sepsis. Only the conventional marker CRP provides certain added value to the iSOFA score for sepsis prediction.
Further investigations should be performed to study the possible diagnostic value of dynamic changes of the three examined markers in prediction and early diagnosis of sepsis.

Keywords: Sepsis, SOFA, presepsin, procalcitonin, abdominal surgery

\section{INTRODUCTION}

Sepsis and septic shock in abdominal surgical patients develop as a consequence of intra-abdominal (IAI) or surgical site infections and can be termed abdominal sepsis. In emergency surgical patients, morbidity and mortality are higher than in non-emergency surgical patients (1). The major risk factors for development of sepsis and septic shock and consequent deaths are age, emergency surgery, presence of different comorbidities and gender (male) (2). According to the Third International Consensus definition of sepsis and septic shock (Sepsis-3), sepsis diagnosis is made when there is an acute change in the total SOFA score of $\geq 2$ points consequent to infection, and patients with septic shock are diagnosed by using a clinical construct of sepsis with persisting hypotension that requires vasopressors to maintain mean arterial pressure (MAP) $65 \mathrm{mmHg}$; also, septic shock patients have a serum lactate level $>2 \mathrm{mmol} / \mathrm{L}$ despite adequate volume resuscitation (3). As the key to successful sepsis treatment lies in the early recognition followed by appropriate therapy and infection source control, early identification of sepsis is challenging and the identi- fication of patients at highest risk for sepsis development is very important $(4,5)$.

The diagnosis of infection is confirmed by microbiological findings that, however, have some serious limitations: they are time-consuming and can be false negative. Therefore, biochemical markers of infection/inflammation could help to not only detect an infection but to detect a failed source control. The traditionally used biomarkers of infection/inflammation are C-reactive protein (CRP), procalcitonin (PCT) and, in recent time, presepsin (PSEP).

This study was conducted in order to examine whether the addition of biomarkers of inflammation/infection PSEP, PCT and CRP to the initial SOFA (iSOFA) scoring system can improve diagnostic accuracy of early sepsis diagnosis in emergency abdominal surgery patients. The specific aims were as follows:

a) To determine the cut-off values of PSEP, PCT and CRP whose sensitivity to sepsis after adding to the iSOFA scoring system is $\geq 95 \%$ and specificity is the highest possible with such sensitivity rate.

b) To determine regression coefficients for the three markers in order to make their diagnostic value highest after the addition to the iSOFA score.

\section{MATERIALS AND METHODS}

The study protocol was approved by the University Hospital Centre Zagreb Ethics Committee and the Faculty of Pharmacy and Biochemistry Ethics Committee. Patients gave their written informed consent 
for participation in the study. The study was designed and executed in accordance with World Medical Association Declaration of Helsinki 2013.

\section{Study population}

We performed a unicentric, prospective, cross-sectional, applied study. The required sample size of 72 (36 septic and 36 non-septic) patients was calculated using data from Yang Y et al. (6). The sample size was calculated using PASS 14 Power Analysis and Sample Size Software (2015), NCSS, LLC. Kaysville, Utah, USA, ncss. com/software/pass. To include 36 abdominal septic patients required for statistical analysis, 244 samples of abdominal emergency patients admitted postoperatively to the 13-bed general surgical intensive care unit (ICU), University Hospital Centre Zagreb, were collected from October 2015 to April 2017. The criteria for enrolment were the following: age between 50 and 80 , acute abdomen diagnosis caused by gastrointestinal inflammatory processes, intestinal obstructions and ileus, dehiscence of a surgical wound, gastrointestinal anastomosis or gastrointestinal perforation. The exclusion criteria were immunosuppressive therapy, and hemato-oncology or general oncology patients with ongoing chemotherapy because of high possibility of immune modulation. The admission to the ICU depended on comorbidity, initial surgical diagnosis, complexity of surgical procedure, intraoperative findings, hemodynamic stability during surgical procedure and general condition of a patient. Before surgery, standard antimicrobial prophylaxis (combination of antibiotics metronidazol and cefazolin or metronidazol and ciprofloxacin) was administered to all patients.

\section{Outcome}

Patients were classified according to the endpoint, i.e. sepsis. The frequent occurrence of primary sepsis in patients undergoing surgical treatment emphasizes the importance of evaluation within first five days. The maximum of five days was chosen because the median ICU length of stay (LOS) for septic and non-septic patients was six (IQR 3-12) and two (IQR 1-4) days, respectively. Sepsis was diagnosed on the basis of the third consensus definition for sepsis and septic shock plus the presence of either documented or suspected infection (3). Suspected infection means that a patient had either negative microbiological tests or there were no microbiological tests requested, but there were clinical signs present such as fever, positive radiological findings, intra-abdominal presence of pus and cloudy fluid, gut perforation and peritonitis stercoralis during surgical procedure.

According to the criteria mentioned above, two independent study physicians classified patients into the following two groups: 1. 36 patients with abdominal sepsis (SE)

2. 36 patients without abdominal sepsis (NSE) with or without infection.

\section{Sampling, laboratory analysis and SOFA calculation}

Lithium-heparin blood samples were collected 12 hours after surgery for CRP, PSEP and PCT determination. For diagnosis of sepsis and septic shock, the SOFA score was calculated perioperatively (iSOFA) and every 24 hours postoperatively during five or less postoperative days depending on patient condition and ICU length of stay. The $\Delta$-SOFA score was the difference between the highest/lowest 24-hour score and admission score. The clinical and laboratory parameters for the SOFA calculation were determined each day as part of morning routine assessment using the SOFA calculator (7). Infection was confirmed by microbiological examination of samples from the sources that should be physiologically sterile (abdominal intraoperative specimen and hemoculture) and/or surgical wound samples. According to the clinical status of a patient and attending surgeon's estimate, microbiological examination was not performed for low-risk patients. PSEP was measured by using the PATHFAST PSEP kit based on chemiluminescent enzyme immunoassay on the PATHFAST Immunoanalyser. PCT was determined by the ELECSYS+ BRAHMS+ PCT assay based on electrochemiluminescent immunoassay and CRP was measured by immunoturbidimetry on Cobas ce6000 analyser.

\section{Statistical analysis}

Statistical significance was determined at $\mathrm{P}<0.05$ level. The hypothesis that a combination of PSEP, PCT, CRP and SOFA scoring system will have a better diagnostic accuracy for the early sepsis recognition than the SOFA score alone was checked by one-tail Spearman 's correlation. Deviation from Gaussian distribution was tested by Shapiro-Wilk test. Nonparametric data were expressed as median and interquartile range (25th-75thIQR). The MannWhitney test was used as nonparametric test for comparison of continuous variables. Diagnostic accuracy was expressed as sensitivity, specificity, area under the receiver operating curve (ROC) and positive and negative likelihood ratios. The cut-off values were determined at $95 \%$ sensitivity and the highest possible specificity with this sensitivity rate. Multivariate logistic regression was used to determine regression coefficients according to presence or absence of sepsis as a dependent variable. The compatibility of the final model with empirical data was checked by the Hosmer-Lemeshow test, and its statistical significance by Omnibus test. The total value of the model was expressed by the Nagelkerke pseudo coefficient of determination (R2) and the total proportion of correctly diagnosed patients. The relationship between PSEP and kidney function parameters, estimated glomerular filtration rate (eGFR) was determined by two-tailed Spearman 's correlation. Statistical analysis was performed using the SPSS statistical program, version 25 (SPSS Inc, Chicago, IL, USA).

\section{RESULTS}

\section{Characteristics of patient population and microbiological findings}

Study subjects were fairly homogenous and all had diagnosis of acute abdomen due to gastrointestinal disturbances. Study patients' data are shown in Table 1. In the SE and NSE, the median (min to max) age was 69 (56-79) and 70 (53-80), respectively. There were no statistical differences between the number of male and female subjects in the two groups (22 vs 21 , respectively, $\mathrm{p}=1.000$ ). Based on the two most frequently established diagnoses, the two groups differed statistically significantly. Perforation/dehiscence diagnosis had 22 septic and 9 non-septic patients. Ileus diagnosis had 10 septic and 16 nonseptic patients, $\mathrm{p}=0.018$. Microbiological studies showed that in the NSE there were negative microbiological findings in 9/18 patients, while no microbiological examinations were performed in 18/36 patients. In the SE group, no microbiological examinations were performed in 2/36 patients and microbiological examinations were negative in 3/34 patients. Verified infections were mostly caused by both gram-positive and gram-negative bacteria $(21 / 34)$. The length of stay in the intensive care unit was more pronounced in septic patients than in NSE patients (6 versus 2 days, $\mathrm{p}<0.001)$. There was no statistical 
Table1. Characteristics of 72 studied patients

\begin{tabular}{|c|c|c|c|}
\hline & SEPTIC & NON-SEPTIC & Statistical significance $(P)$ \\
\hline $\mathrm{N}$ (number of patients) & $36 / 7^{a}$ & 36 & \\
\hline Age & $69(56-79)$ & $70(53-80)$ & 0.874 \\
\hline \multicolumn{4}{|l|}{ Gender } \\
\hline Male & 22 & 21 & 1.000 \\
\hline Female & 14 & 15 & 1.000 \\
\hline \multicolumn{4}{|l|}{ Diagnosis } \\
\hline Ileus & 10 & 16 & $0.018^{\star}$ \\
\hline Perforation, dehiscence & 22 & 9 & $0.018^{\star}$ \\
\hline Peritonitis & 1 & 1 & \\
\hline Hernia incarcerate & 1 & 3 & \\
\hline Gangrene & 1 & 1 & \\
\hline Cholecystitis acuta & 1 & 4 & \\
\hline Pancreatitis acuta & 0 & 1 & \\
\hline Pancolitis & 0 & 1 & \\
\hline Infection & $34 / 36$ & $18 / 36$ & \\
\hline \multicolumn{4}{|l|}{$\begin{array}{l}\text { Microbiological analysis } \\
\text { requested }\end{array}$} \\
\hline Negative & $3 / 34$ & $9 / 18$ & \\
\hline Gram negative and positive & $21 / 34$ & $5 / 18$ & \\
\hline Gram positive & $8 / 34$ & $4 / 18$ & \\
\hline Gram negative & $2 / 34$ & $0 / 18$ & \\
\hline ICU LOS (days) & $6\left(1-125^{b} / 3-12^{c}\right)$ & $2\left(1-8^{b} / 1-4^{c}\right)$ & $<0.001^{*}$ \\
\hline Outcome (28-day mortality) & & & 0.055 \\
\hline YES & 7 & $1^{\mathrm{d}}$ & \\
\hline $\mathrm{NO}$ & 29 & 35 & \\
\hline
\end{tabular}

a Septic shock developed in seven patients

b Minimum and maximum value

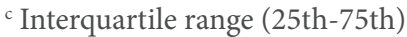

d Lethal outcome caused by cardiorespiratory arrest; not infection- nor sepsis-related

Mann-Whitney test was used to examine statistically significant difference between two groups according to age and ICU LOS, and Fisher's exact test according to gender, clinical diagnosis and outcome.

Table 2. Type of sample taken for microbiological analysis

\begin{tabular}{lll}
\hline & SEPSIS & YES \\
\hline 0-Non-analyzed & 18 & 2 \\
\hline 1-Intraoperative specimen & 6 & 20 \\
\hline 2-Hemoculture & 0 & 7 \\
\hline 3-Surgical swab & 2 & 5 \\
\hline 4-Minilavate & 1 & 1 \\
\hline 5-Tracheal aspirate & 4 & 1 \\
\hline 6-Surgical drain & 1 & 0 \\
\hline 7-Other swab & 4 & 0 \\
\hline
\end{tabular}

difference in lethal outcomes on day 28 between SE and NSE patients (7/36 and $1 / 36$, respectively, $\mathrm{p}=0.055)$. Table 2 shows the sites from which samples were taken for microbiological analysis. One lethal outcome in the NSE group was caused by cardiorespiratory arrest; there was no infection or sepsis.

\section{Comparison of the iSOFA score and three biochemical markers between the two groups of patients}

The median and IQR for four parameters of the two patient groups are shown in Table 3, Figure 1 (A-D). The Mann-Whitney test showed significant differences between
SE and NSE group in the iSOFA score, PSEP and PCT [SE 10(IQR 8-12) and NSE 1(IQR 1-2), p<0.001; SE 1121.5(IQR 462.52265.0) and NSE 525.5(IQR 269.8-696.0), $\mathrm{p}<0.001$; SE 2.96(IQR 1.80-23.78) and NSE 0.75(IQR 0.30-9.30, p=0.005], respectively. There was no significant difference in CRP (SE 151.1(IQR 63.4-282.3) and NSE 101.4(IQR 36.5-225.9), $\mathrm{p}=0.120$ ). In the NSE group, there was one extreme outlier in PSEP measurement (11089 ng/L). This result was not removed from statistical analysis because it was not the measurement error and there was no difference in statistical analysis when this case was excluded.

\section{Diagnostic accuracy and cut-off values of PSEP, PCT and CRP combined with the iSOFA score}

One-tailed Spearman's correlation for verification of the main hypothesis shows that there is a statistically significant cor- 
Table 3. The median and 25th-75th IQR for four parameters in the two groups of patients

\begin{tabular}{|c|c|c|c|c|c|c|c|}
\hline Parameters & SEPSIS & $\mathbf{N}$ & Median & Interquartile range & Minimum & Maximum & $\mathbf{P}$ \\
\hline \multirow[t]{2}{*}{ iSOFA } & No & 36 & 1 & $1-2$ & 0 & 7 & $<0.001^{\star}$ \\
\hline & Yes & 36 & 10 & $8-12$ & 1 & 16 & \\
\hline \multirow[t]{2}{*}{ PSEP } & No & 36 & 525.5 & $269.8-696.0$ & 111.0 & 11089.01 & $<0.001^{\star}$ \\
\hline & Yes & 36 & 1121.5 & $462.5-2265.0$ & 342.0 & 7873.0 & \\
\hline \multirow[t]{2}{*}{ PCT } & No & 36 & 0.75 & $0.30-9.30$ & 0.08 & 38.2 & $0.005^{*}$ \\
\hline & Yes & 36 & 2.96 & $1.80-23.78$ & 0.14 & 100.0 & \\
\hline \multirow[t]{2}{*}{ CRP } & No & 36 & 101.4 & $36.5-225.9$ & 2.4 & 450.0 & 0.120 \\
\hline & Yes & 36 & 151.1 & $63.4-282.3$ & 14.6 & 515.5 & \\
\hline
\end{tabular}

*Mann-Whitney test

iSOFA - initial sequential organ failure assessment

PSEP - presepsin

PCT - procalcitonin

CRP - C-reactive protein

Table 4. Diagnostic accuracy of four markers for sepsis diagnosis

\begin{tabular}{lllllllll}
\hline & AUC & $\mathbf{P}$ & $\begin{array}{l}\text { 95\% Confidence } \\
\text { Interval }\end{array}$ & Cut-off value & Sensitivity & Specificity & $\begin{array}{l}\text { Positive } \\
\text { likelihood ratio }\end{array}$ & $\begin{array}{l}\text { Negative } \\
\text { likelihood ratio }\end{array}$ \\
\hline iSOFA & 0.967 & $<0.001^{*}$ & $0.925-1.000$ & 2 point & 0.972 & 0.528 & 2.051 & 0.053 \\
\hline PSEP & 0.738 & $0.001^{*}$ & $0.623-0.853$ & 349 & 0.972 & 0.333 & 1.460 & 0.084 \\
\hline PCT & 0.694 & $0.005^{*}$ & $0.571-0.817$ & 0.17 & 0.972 & 0.167 & 1.167 & 0.168 \\
\hline CRP & 0.606 & 0.120 & $0.476-0.737$ & 18.1 & 0.972 & 0.139 & 1.129 & 0.201 \\
\hline
\end{tabular}

${ }^{*} \mathrm{ROC}$ analysis

iSOFA - initial sequential srgan failure assessment

PSEP - presepsin

PCT - procalcitonin

CRP - C-reactive protein

relation between the iSOFA score and two markers, PSEP and PCT (Spearman's rho 0.363, $\mathrm{p}=0.001$ and Spearman's rho was $0.354, p<0.001)$. There was no significant correlation between the iSOFA score and CRP, $\mathrm{p}=0.239$, as shown in Table 4 . ROC analysis was performed to assess diagnostic accuracy for four parameters. The results showed that the AUC value for the iSOFA was highest, followed by the AUC value obtained for PSEP, PCT and CRP [0.967 (95\% CI 0.925-1.000), $\mathrm{p}<0.001 ; 0.738$ (95\% CI $0.623-0.853), \mathrm{p}=0.001 ; 0.694$ (95\% CI $0.571-0.817), \quad \mathrm{p}=0.005 ; 0.606 \quad(95 \% \quad \mathrm{CI}$ $0.476-0.737), \mathrm{p}=0.120]$, respectively. The cut-off values of SOFA, PSEP and PCT for diagnosing sepsis with $95 \%$ sensitivity and the highest possible specificity with such sensitivity rate were 2 points, $349 \mathrm{ng} / \mathrm{L}$ and $0.17 \mu \mathrm{g} / \mathrm{L}$, respectively (Table 4 and Figure 2).

\section{Multiple logistic regression analysis of the iSOFA score by adding PSEP, PCT and CRP}

Logistic regression analysis was performed to assess the impact of markers on the likelihood that a patient has sepsis. There are two models: the first contained one independent variable (iSOFA score) and the second contained four independent variables (iSOFA score, PSEP, PCT and Creactive protein). The first and the second model were both statistically significant, $\chi 2$ $(1, \mathrm{~N}=72)=70.5, \mathrm{p}<0.001$ and $\chi 2(4, \mathrm{~N}=72)$ $=81.4, \mathrm{p}<0.001$, respectively, indicating that the models are able to distinguish SE from NSE. The second model was better in discriminating the two groups of patients. Hosmer and Lemeshow test also supports both models as reliable, first model $\chi^{2}$ (6) $=1.27, \mathrm{p}=0.974$ and second model $\chi 2(6)=$ 2.46, $\mathrm{p}=0.963$. The models explained $83 \%$ and $90 \%$ of the variance of sepsis diagnosis and correctly classified $92 \%$ and $96 \%$ cases, respectively, Table 5 . As shown in Table 6, the SOFA score is the strongest predictor of sepsis and, of the three markers, only CRP contributes statistically significantly to the model. The odds ratio for the SOFA score in the first model is 2.304 (95\% CI $1.583-3.354, \mathrm{p}<0.001)$ and in the second model 3.22 (95\% CI 1.696-6.102, $\mathrm{p}<0.001)$. These results indicate that patients with the higher initial SOFA score without markers are 2.3 times more likely to have sepsis, and patients with the higher initial SOFA score and simultaneously obtained high concentration of three markers are 3.2 times more likely to have sepsis.

The regression equation obtained for the second model is as follows:

SEPSIS $=-8.988+1.17($ SOFA $)+0.015$ (CRP)

\section{Correlation of PSEP and the kidney func- tion parameters}

The results showed statistically significant negative correlation between PSEP and eGFR, Spearman's rho was -0.593 , $\mathrm{p}<0.001$. Coefficient of determination was 0.352 , Figure 3 .

\section{DISCUSSION}

Despite the last Sepsis-3 definition which confirms that changes in the SOFA score form the basis of sepsis diagnosis (3), the routine use of biochemical markers of infection/inflammation are nevertheless of great help in the early recognition, risk 
Table 5. Total value of models in distinguishing SE from NSE patients

A)

\begin{tabular}{llllll}
\hline Predicted & & & Percentage Correct & Nagelkerke R Square \\
\hline & & SEPSIS & & \\
\hline SEPSIS & NO & 33 & YES & 31.7 & 0.832 \\
& NO & 3 & 33 & 91.7 & \\
\hline
\end{tabular}

Overall percentage 91.7

B)

\begin{tabular}{|c|c|c|c|c|c|}
\hline \multicolumn{4}{|c|}{ Predicted } & \multirow[t]{2}{*}{ Percentage Correct } & \multirow[t]{2}{*}{ Nagelkerke R Square } \\
\hline & & \multicolumn{2}{|c|}{ SEPSIS } & & \\
\hline & & $\mathrm{NO}$ & YES & & \\
\hline \multirow[t]{2}{*}{ SEPSIS } & $\mathrm{NO}$ & 35 & 1 & 97.2 & 0.903 \\
\hline & YES & 2 & 34 & 94.4 & \\
\hline
\end{tabular}

Overall percentage 95.8

Logistic regression: A) First model is based only on the initial SOFA score, B) Second model takes into account all four markers

Table 6. Logistic regression predicting likelihood of sepsis

\begin{tabular}{llllll}
\hline & & & & \multicolumn{2}{c}{$\mathbf{9 5 \%}$ Confidence interval for OR } \\
\hline FIRST MODEL & $\mathrm{B}$ & $\mathrm{p}$ & $\mathrm{OR}$ & Lower & Upper \\
\hline iSOFA & 0.835 & $<0.001^{\star}$ & 2.304 & 1.583 & 3.354 \\
\hline & & & & & Upper \\
\hline SECOND MODEL & $\mathrm{B}$ & $\mathrm{p}$ & $\mathrm{OR}$ & 1.696 & 6.102 \\
\hline iSOFA & 1.168 & $<0.001^{*}$ & 3.217 & 1.000 & 1.001 \\
\hline PSEP & 0.000 & 0.225 & 3.217 & 0.925 & 1.046 \\
\hline PCT & -0.016 & 0.605 & 0.984 & 1.003 & 1.028
\end{tabular}

iSOFA - initial sequential organ failure assessment

PSEP - presepsin

PCT - procalcitonin

CRP - C-reactive protein

stratification and therapy monitoring. The principal aim of this study was to investigate whether the well-established routine markers PCT and CRP (8), along with the newly introduced PSEP, could provide added value, together with the initial SOFA score, for the prediction and early diagnosis of sepsis in emergency abdominal surgery patients admitted to the general surgical ICU. In accordance to our specific aim the initial SOFA score was used as a helping tool for early sepsis diagnosis despite defined SOFA score changes as a parameter on which the diagnosis of sepsis is based (3).

As the early identification of patients at risk for sepsis development is crucial in order to confirm and remove the infectious source as soon as possible, a broad spectrum of antimicrobial therapy should be administered to stop spreading the infection $(4,9,10)$. In this study we measured perioperative PSEP, PCT and CRP as markers of infection/inflammation and evaluated their diagnostic accuracy, the accuracy of the initial SOFA score separately and of the combination of all aforementioned parameters for sepsis diagnosis. To the best of our knowledge, previous studies did not investigate these four markers together in the specific population of emergency abdominal surgery patients.

The study revealed that the initial SOFA score, PSEP and PCT were significantly higher in septic than in non-septic group of patients, which is in accordance with previous studies (11-14). The CRP was not significantly different in the two groups of patients, as was already seen in other studies with similar patient population $(15,16)$. Santonocito C et al. reported different results but they studied a different patient population and, unlike our study, examined postoperative CRP changes (17). With regard to our chosen sepsis sensitivity threshold of $\geq 95 \%$, the obtained cut-off value for the iSOFA score was two points. The AUC was 0.967 and specificity $53 \%$. Other studies have not shown so high AUC for the SOFA score $(6,18,19)$ probably because heterogeneous patient populations were involved, as opposed to our study which comprised fairly homogenous patient population. The obtained specificity for iSOFA was pretty low. However, compared with specificity obtained for other three markers it could be concluded that iSOFA was a parameter with the highest specificity for sepsis.

The logistic regression analysis of two models, the first including only the iSOFA score and the second combining three biomarkers and the iSOFA score, showed that 

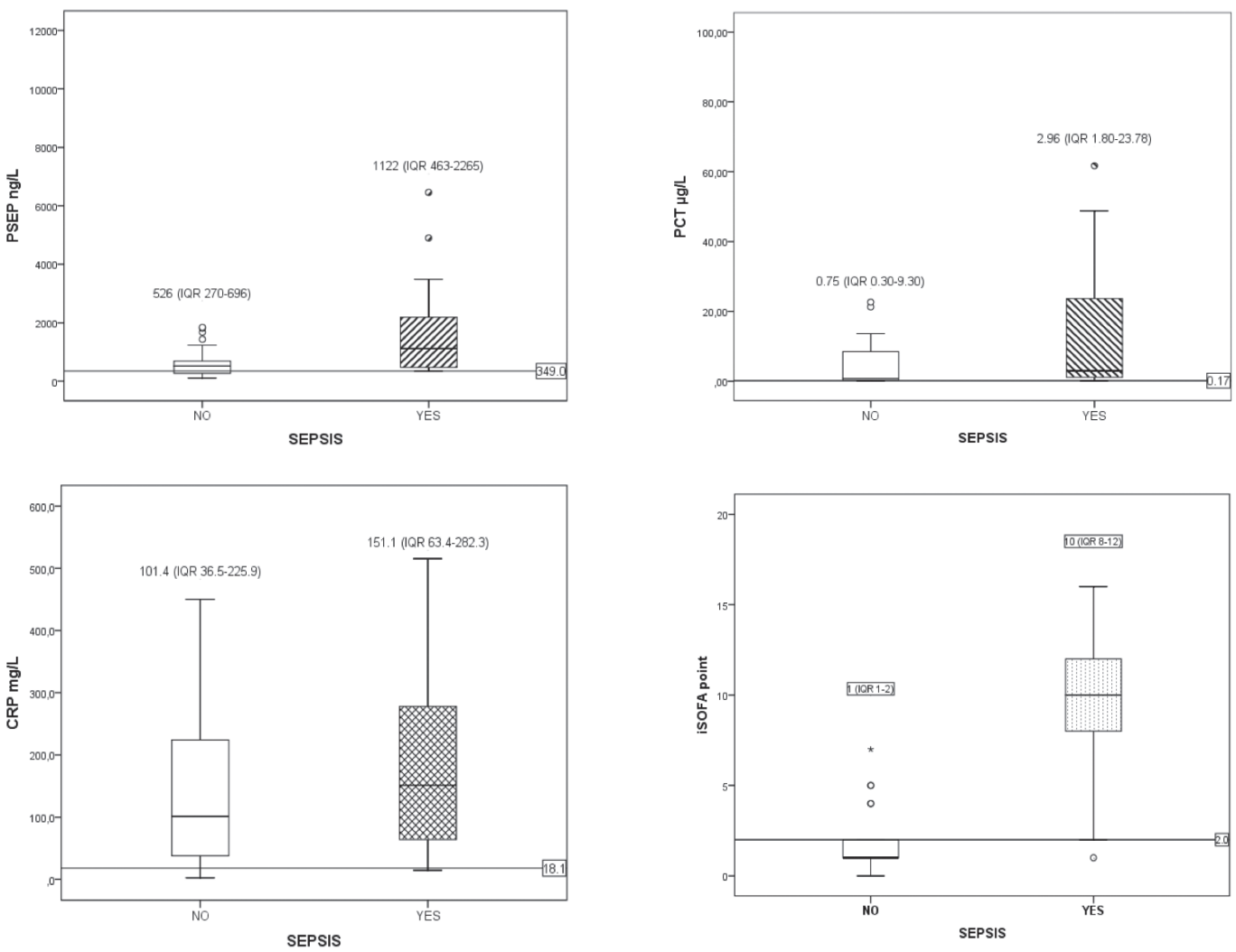

Figure 1. Levels of four markers in SE and NSE group

A) presepsin, B) procalcitonin, C) C-reactive protein and D) initial SOFA score. Lines across inside the box represent median values and whiskers represent minimum and maximum values. The reference lines to the y axis represent cut-off values obtained according to the specific aim.

iSOFA - initial sequential organ failure assessment, PSEP - presepsin, PCT - procalcitonin, CRP - C-reactive protein

-of three biomarkers- only CRP contributed statistically significantly to the diagnostic performance of the model involving only iSOFA score. For the first model, the analysis showed that patients with a higher SOFA score are almost two times more likely to suffer from sepsis. In the second model, patients with a higher iSOFA score and a higher level of biomarkers are two times more likely to have sepsis. The first model was shown to correctly classify $91.7 \%$ patients and the second model $95.8 \%$ patients. This means that, out of one hundred patients, we can diagnose sepsis in four additional patients using the second model with added biochemical markers. The logistic regression for the second model showed that PSEP and PCT do not contribute to the model at all. But CRP, which has by itself been shown as unable to distinguish a septic from non-septic patient, provides additional value for the SOFA score in our model.

Regardless of the fact that the biochemical markers do not contribute statistically significantly to the iSOFA score, we determined the cut-off values of each marker bearing in mind our specific aim. For the desired sensitivity of $\geq 95 \%$, the obtained cut-off values for sepsis were: PSEP 349 $\mathrm{ng} / \mathrm{L}$, PCT $0.17 \mu \mathrm{g} / \mathrm{L}$ and CRP $18.1 \mathrm{mg} / \mathrm{L}$. PSEP showed a slightly higher diagnostic accuracy than PCT (AUC 0.738 vs. 0.694). It was shown that CRP with AUC 0.606 cannot distinguish septic from non-septic patients. Specificity for all three markers PSEP, PCT and CRP was fairly low, i.e. $33 \%, 17 \%$ and $14 \%$, respectively.

The determined cut-off values for PSEP and PCT are near the upper reference limit (20-22). These low cut-off values are the consequence of our specific aim which involved high sensitivity and thus early sepsis recognition. Similar results and a fairly low cut-off value for CRP were obtained by Meyer $\mathrm{ZC}$ et al. when they examined dynamic changes of CRP during the ICU course (23). In this study there was one patient within the control group with an extremely high PSEP value (11.089 ng/L) together with very high PCT and CRP values $(35.64 \mu \mathrm{g} / \mathrm{L}$ and $169.0 \mathrm{mg} / \mathrm{L}$, respectively). Those results were not removed from statistical analysis because there was no question of error in the patient identification and measurement. Unfortunately, the microbiological examination was not done for this patient and we do not know if there was any infection. This patient suffered from liver dysfunction with low cholinesterase activity, renal insufficiency and COPD. A study from Papp et al. (24) showed that PSEP is a useful infection marker in liver disease but in renal failure the PSEP level increases as the glomerular filtration rate (GFR) decreases (24-26). Masson et al. have shown that high levels of PSEP can be found in COPD patients (27). Inflammatory/infection markers can be increased in patients with COPD exacerbation (28) but this was not the case with their patient. The patient was classified in the non-septic group according to the SOFA score (one point), spent only one day in ICU, was discharged from hospital after five days and was alive after 28 days. The suspicion remains that the patient still had infection but it has never been proven. The examined correlation of PSEP with the estimated GFR in this study showed inverse connection. This is the reason why 


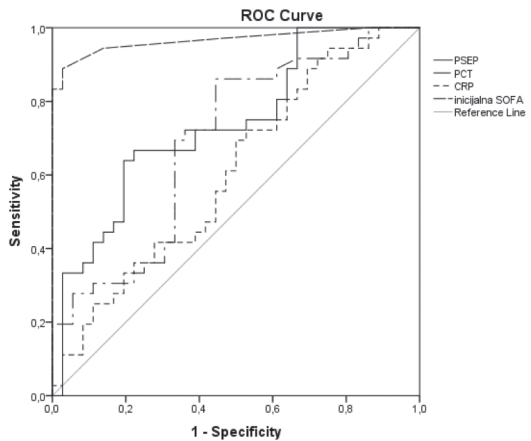

Figure 2. ROC analysis of four markers

iSOFA - initial sequential organ failure assessment

PSEP - presepsin

PCT - procalcitonin

CRP - C-reactive protein

the interpretation of PSEP values must be related to the kidney function.

Our study consists of consecutively included patients as they were admitted postoperatively to the surgical ICU. No healthy subjects were included in the study as a control group, and the markers used (except PSEP) are part of the actual routine practice in the general surgical ICU. Also, although this is a study with small sample size, it was designed to minimize patient heterogeneity.

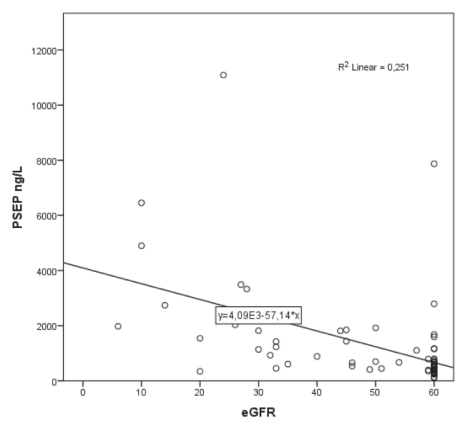

Figure 3. Correlation of PSEP and the eGFR

PSEP - presepsin

eGFR - estimated glomerular filtration rate

\section{LIMITATIONS}

The main limitation of this study, besides relatively small sample size and unicentricity, is the fact that the microbiological examinations were not done in all patients and therefore there are no complete data for each patient about the presence or absence of infection.

\section{CONCLUSION}

The SOFA score is known to be the best diagnostic tool for sepsis diagnosis, but it seems that PSEP and PCT are among the three investigated markers -although not contributing to the iSOFA score- good independent markers with significantly higher levels in septic than in non-septic patients. PSEP has the highest diagnostic accuracy for sepsis. Only the conventional marker CRP provides certain added value to the iSOFA score for sepsis prediction.

Further investigations should be performed to study the possible diagnostic value of dynamic changes of the three examined markers in prediction and early diagnosis of sepsis.

\section{ACKNOWLEDGMENTS}

The authors are very grateful to the employees of The Unit for Emergency Laboratory Diagnostics and POCT, University Hospital Center Zagreb, for help with storage of patient samples. We are grateful to administrative staff of the Department of Anesthesiology and ICU, University Hospital Centre Zagreb, for their collaboration regarding access to treatment protocols. We are also grateful to Nikola Habuzin for providing language assistance.

\section{REFERENCES}

3. Havens JM, Peetz AB, Do WS, Cooper Z, Kelly E, Askari R, et al. The excess morbidity and mortality of emergency general surgery. J Trauma Acute Care Surg 2015;78(2):306-11.

4. Moore LJ, Jones SL, Xu J, Bass BL. Sepsis in general surgery: a deadly complication. Am J Surg 2009;198(6):868-74.

5. Singer M, Deutschman CS, Seymour CW, Shankar-Hari M, Annane D, Bauer M, et al. The Third International Consensus Definitions for Sepsis and Septic Shock (Sepsis-3). JAMA 2016;315(8):801-10.

6. Mazuski JE, Tessier JM, May AK, Sawyer RG, Nadler EP, Rosengart MR, et al. The Surgical Infection Society Revised Guidelines on the Management of Intra-Abdominal Infection 2017;18(1):1-76

7. Moore LJ, Sepsis in General Surgery. Arch Surg 2010;145(7):695.

8. Yang Y, Xie J, Guo F, Longhini F, Gao Z, Huang Y, et al. Combination of C-reactive protein, procalcitonin and sepsis-related organ failure score for the diagnosis of sepsis in critical patients. Ann Intensive Care 2016;6(1):51.

9. Sequential Organ Failure Assessment (SOFA) Calculator - ClinCalc.com.

10. Pierrakos C, Vincent J-L. Sepsis biomarkers: a review. Crit Care 2010;14(1):R15.

11. Dellinger RP, Schorr CA, Levy MM. A user`s guide to the 2016 Surviving Sepsis Guidelines. Intensive Care Med2017;43(3):299-303..

12. Rhodes A, Evans LE, Alhazzani W, Levy MM, Antonelli M, Ferrer R, et al. Surviving Sepsis Campaign: International Guidelines for Management of Sepsis and Septic Shock: 2016. Intensive Care Med 2017;43(3) :304-377.

13. Vodnik T, Kaljevic G, Tadic T, Majkic-Singh N. Presepsin (sCD14-ST) in preoperative diagnosis of abdominal sepsis. Clin Chem Lab Med 2013;51(10):2053-62.

14. Yaegashi Y, Sato N, Suzuki Y, Kojika M, Imai S, Takahashi G, et al. Evaluation of a newly identified soluble CD14 subtype as a marker for sepsis. J Infect Chemother 2005;11(5):234-8.

15.Zhang X, Liu D, Liu Y-N, Wang R, Xie L-X. The accuracy of presepsin (sCD14-ST) for the diagnosis of sepsis in adults: a metaanalysis. Crit Care 2015;19:323.

16. Pupelis G, Drozdova N, Mukans M, Malbrain ML. Serum procalcitonin is a sensitive marker for septic shock and mortality in secondary peritonitis. Anestezjol Intens Ter 2014;46(4):262-73.

17. Ravishankaran P, Shah AM, Bhat R. Correlation of Interleukin-6, Serum Lactate, and C-Reactive Protein to inflammation, complication, and outcome during the surgical course of patients with acute abdomen. J Interf Cytokine Res 2011;31(9):685-90. 
18. Domínguez-Comesaña E, López-Gómez V, Estevez-Fernández SM, Mariño Padín E, Ballinas-Miranda J, Carrera-Dacosta E, et al. Procalcitonin and C-Reactive Protein as early indicators of iostoperative intra-abdominal infection after surgery for gastrointestinal cancer. Cirugía Española 2014;92(4):240-6.

19. Santonocito C, De Loecker I, Donadello K, Moussa MD, Markowicz S, Gullo A, et al. C-reactive protein kinetics after major surgery. Anesth Analg 2014;119(3):624-9.

20. Wu J, Hu L, Zhang G, Wu F, He T. Accuracy of presepsin in sepsis diagnosis: a systematic review and meta-analysis. Groeneveld ABJ, editor. PLoS One 2015;10(7):e0133057.

21. Gans SL, Atema JJ, Van Dieren S, Bas \&, Koerkamp G, Boermeester MA. Diagnostic value of C-reactive protein to rule out infectious complications after major abdominal surgery: a systematic review and meta-analysis 2015;30(7)851-73

22. Giavarina D, Carta M. Determination of reference interval for presepsin, an early marker for sepsis. Biochem medica 2015;25(1):648.

23. Shozushima T, Takahashi G, Matsumoto N, Kojika M, Okamura Y, Endo S. Usefulness of presepsin (sCD14-ST) measurements as a marker for the diagnosis and severity of sepsis that satisfied diagnostic criteria of systemic inflammatory response syndrome. J Infect Chemother 2011;17(6):764-9.

24. Riedel S, Melendez JH, An AT, Rosenbaum JE, Zenilman JM. Procalcitonin as a marker for the detection of bacteremia and sepsis in the emergency department. Am J Clin Pathol 2011;135(2):182-9.

25. Meyer ZC, Schreinemakers JMJ, Mulder PGH, de Waal RAL, Ermens AAM, van der Laan L. The role of C-Reactive Protein and the SOFA Score as parameter for clinical decision making in surgical patients during the intensive care unit course. Salluh JIF, editor. PLoS One 2013;8(2):e55964.

26. Papp M, Tornai T, Vitalis Z, Tornai I, Tornai D, Dinya T, et al. Presepsin teardown - pitfalls of biomarkers in the diagnosis and prognosis of bacterial infection in cirrhosis. World J Gastroenterol 2016;22(41):9172-85.

27. Kotera A, Sagishima K, Tashiro T, Niimori D, Kamohara H, Kinoshita Y. A validation of presepsin levels in kidney dysfunction patients: four case reports. J Intensive Care 2014;2(1):63.

28. Nagata T, Yasuda Y, Ando M, Abe T, Katsuno T, Kato S, et al. Clinical impact of kidney function on presepsin levels. PLoS One 2015;10(6):e0129159.

29. Masson S, Caironi P, Fanizza C, Thomae R, Bernasconi R, Noto A, et al. Circulating presepsin (soluble CD14 subtype) as a marker of host response in patients with severe sepsis or septic shock: data from the multicenter, randomized ALBIOS trial. Intensive Care Med 2015;41(1):12-20.

30. Lacoma A, Prat C, Andreo F, Lores L, Ruiz-Manzano J, Ausina V, et al. Value of procalcitonin, C-reactive protein, and neopterin in exacerbations of chronic obstructive pulmonary disease. Int J Chron Obstruct Pulmon Dis 2011;6:157-69. 